\title{
Hyperhidrosis treatment with bornaprine in the acute phase of spinal cord-injured patients
}

\author{
R Sergi, A Massone, S Moretto, C Oggerino, F Bertolotto, L Losio and M Ottonello \\ Department of Rehabilitation, Spinal Cord Injury Unit, Santa Corona Hospital, Pietra Ligure, Savona, Italy
}

\begin{abstract}
Study design: Original article.
Objective: To test the use of bornaprine in the hyperhidrosis treatment in the acute phase of spinal cord-injured patients.

Setting: Patients with acute spinal cord lesions in the Spinal Unit of Pietra Ligure, Savona, Italy.

Method: In 4 years, 12 patients have been treated, for a maximum period of 75 days, with dosages ranging from 2 to $4 \mathrm{mg}_{\text {day }}{ }^{-1}$.

Results: All of them reported long-lasting subjective benefits, without side effects, even after the interruption of the therapy.

Conclusion: The bornaprine treatment, at 2 or $4 \mathrm{mgday}^{-1}$ dosage, has been effective and safe to contrast hyperhidrosis, in the acute phase of spinal cord-injured patients. This positive experience would require additional trials and a larger number of cases to gain a more solid support.

Spinal Cord (2008) 46, 571-573; doi:10.1038/sc.2008.12; published online 11 March 2008
\end{abstract}

Keywords: bornaprine; hyperhidrosis; spinal cord injury

\section{Introduction}

Thermoregulation problems are accounted among the serious health disorders, subsequent to spinal cord injuries. Hypohidrosis or hyperhidrosis occurrences are quite frequent. The former usually does not bother much, whereas the latter creates a great deal of discomfort in the patient.

Several therapeutic options are available to treat hyperhidrosis. Oral medications, epidural alcoholic block, sympathectomy and surgical ablation of the sweat glands are some of them.

A number of molecules have been tested successfully. ${ }^{1-4}$ Unfortunately, no oral medication is specifically authorized for hyperhidrosis by the Italian Health Department.

We decided to treat in-patients affected by intolerable hyperhidrosis symptoms with bornaprine, on the basis of its reported effectiveness ${ }^{5}$ and also the lack of effective noninvasive alternative.

\section{Methods}

From August 2002 to July 2006, a 4-year trial has been carried out. Twelve spinal cord-injured patients (10 men and 2

Correspondence: Dr R Sergi, Unità Spinale Unipolare, Ospedale Santa Corona, Via XXV Aprile 38, 17027 Pietra Ligure, Savona, Italy.

E-mail: roberto.sergi@ospedalesantacorona.it

Received 20 September 2007; revised 27 December 2007; accepted 13 January 2008; published online 11 March 2008 women; age range 18-67 years, average 42 years) have been treated with bornaprine for hyperhidrosis in their first 4 months from the injury, which they reported subjectively distressing.

All patients presented a traumatic medullary lesion. All had been surgically treated: six at the vertebral surgical ward in our hospital and six in neurosurgery departments from other centres.

Eight patients showed American Spinal Injury Association (ASIA) impairment scale ' $A$ ' and four patients ASIA impairment scale ' $\mathrm{B}$ '. The cervical lesions were three (quadriplegic), whereas the dorsal lesions were nine (paraplegic) (Figure 1).

The 12 patients were treated with one or more of these drugs: low-molecular-weight heparin, baclofen, oxybutynin, ciprofloxacin, amoxicillin/clavulanic acid, amikacin.

They suffered from hyperhidrosis not only at night but during the day as well, with bouts of sweating.

Eight in-patients presented one or more episodes of urinary tract infection, treated with antibiotics based on antibiogram, for a period varying from 8 to 10 days.

Only two patients presented fever $<38^{\circ} \mathrm{C}$ for $3-4$ days without leukocytosis. All the patients suffered from sweating before the setting in of the infection, as well as during and after it, while on antibiotics and in absence of antibiotic treatment.

For these reasons, hyperhidrosis has not been attributed to the septic state, nor to the antibiotic treatment. 

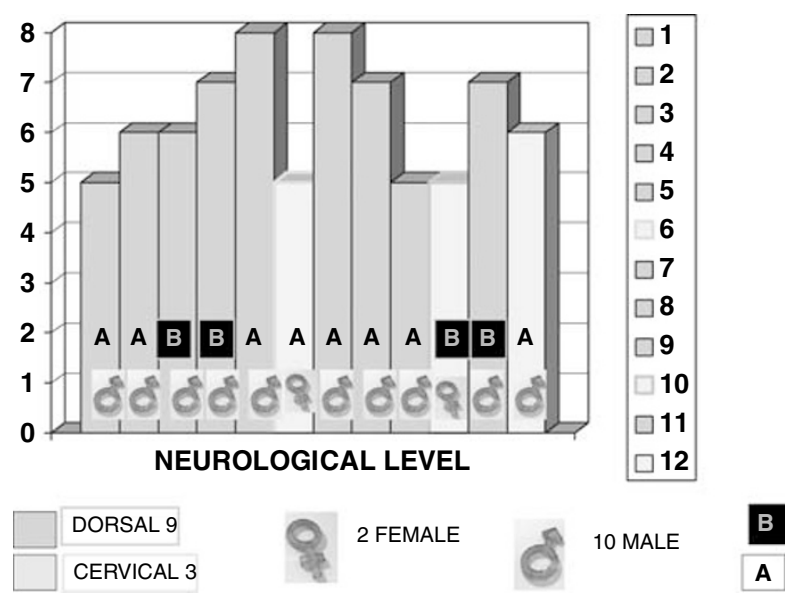

Figure 1 Neurological level and impairment scale American Spinal Injury Association of patients.

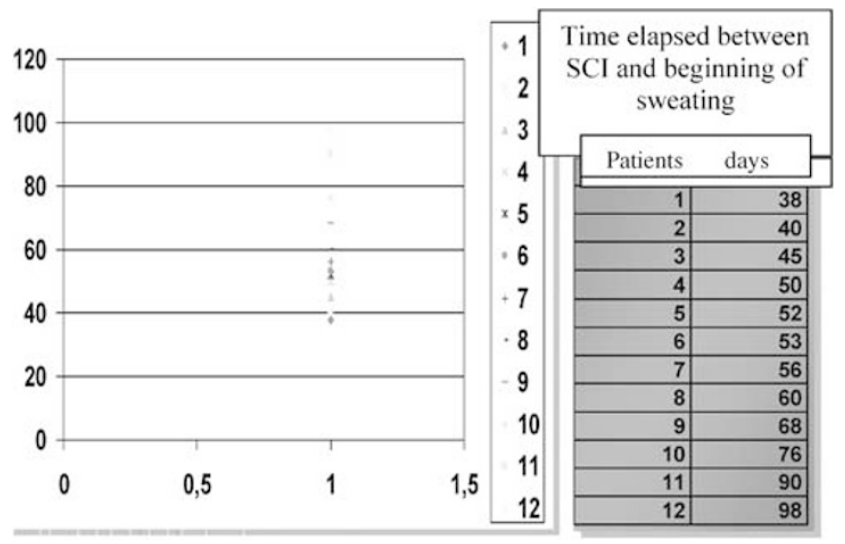

Figure 2 Time elapsed between spinal cord injury and beginning of sweating.
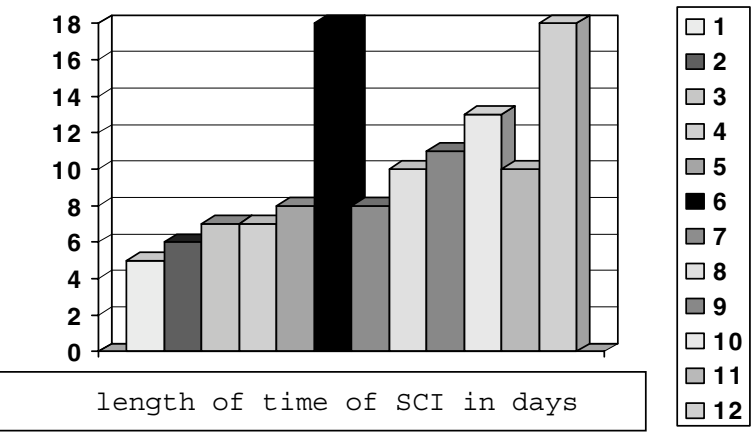

Figure 3 Length of time of spinal cord lesion.

During hospitalization, $9 / 12$ patients (75\% of the cohort) advanced from the flaccid phase to the spastic one. The spastic phase developed different grades of seriousness leading in some cases to the use of baclofen orally. Although, we do not believe there is a link between spasticity and hyperhidrosis.

Two patients (man, T5 'A' and woman, C6 'B') presented first two episodes and the latter one episode of autonomic

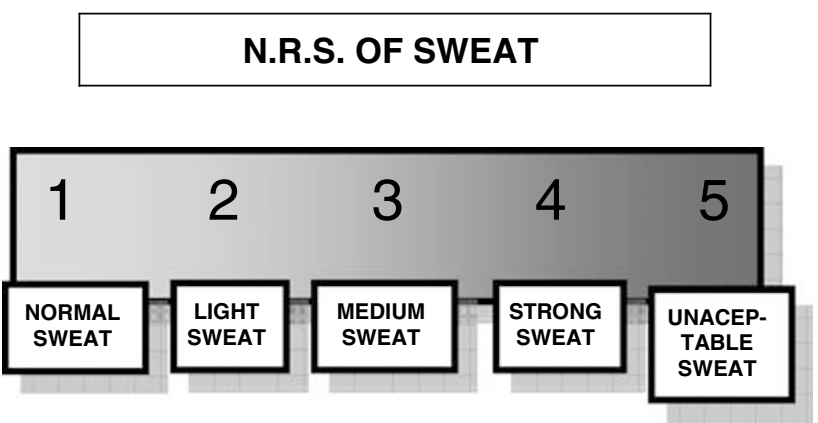

Figure 4 Numeric rating scale of sweat.

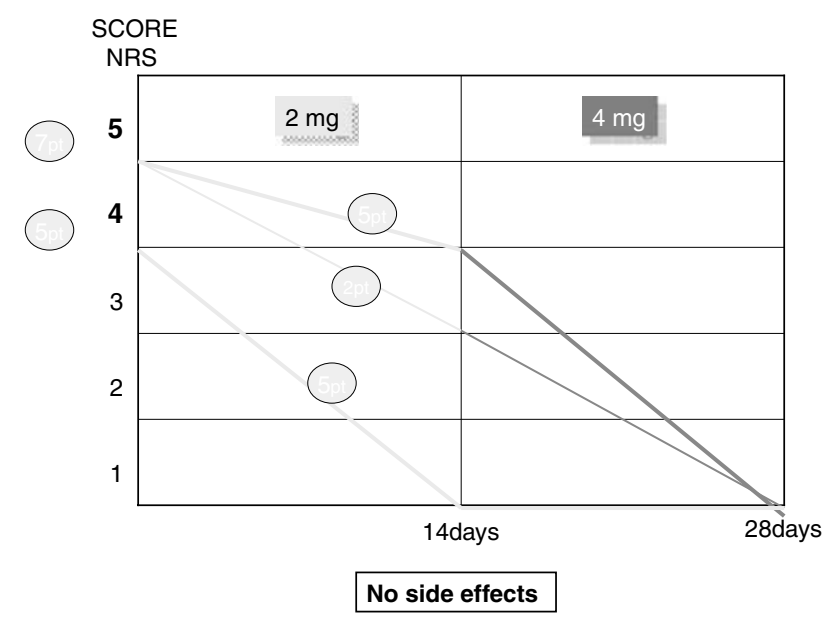

Figure 5 Effects of therapy.

dysreflexia during hospitalization. In the first case, the event had been provoked by the obstruction of the indwelling catheter, whereas the second episode had been caused by the presence of fecaloma. Both cases had been localized and lasted for a few minutes. The same patients had been suffering from hyperhidrosis for many days before the above-mentioned troubles set in.

Causes of hyperhidrosis, such as hyperthyroidism and other endocrine disorders, hormonal treatment of prostate cancer or other types of malignant tumours, mental illness and idiopathic hyperhidrosis, have been excluded.

Excessive sweating started between the 38th and the 98th day from the spinal cord injury (Figure 2).

All patients were hospitalized in our centre. We hospitalized patients as quickly as possible from the time of the injury, as soon as they were discharged from the intensive care or from the surgery department where they had been treated (Figure 3).

Before starting the therapy, a numeric rating scale (NRS) test was run with the following measurement criteria (Figure 4):

score 1: normal sweat;

score 2: slight sweat increase above the normal; score 3: medium sweat increase above the normal; score 4: high sweat increase above the normal; and score 5: highly disturbing and unacceptable sweat levels. 


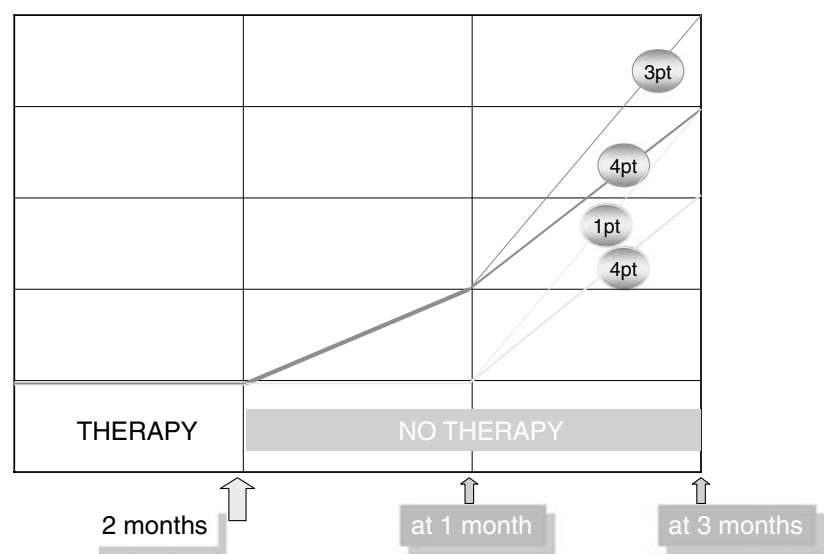

Figure 6 Results after suspension of the therapy.

At the beginning, a $2 \mathrm{mg} \mathrm{day}^{-1}$ dose was administered to all the patients. For those patients with a poor response to the therapy, the dosage was increased by $2 \mathrm{mg}$, for every 2 weeks. The treatment continued for 2 months after the response to the therapy occurred. A follow-up NRS test was performed after 1 and 3 months from the therapy interruption.

A written consent before starting the treatment has became necessary, as, in Italy, bornaprine is authorized for specific usage with Parkinson's syndrome only, especially when tremor symptoms are prevalent.

\section{Results}

The initial NRS score for the 12 patients was as follows: 9 patients, score $4 ; 3$ patients, score 5 .

For five patients, an administered dose of $2 \mathrm{mg} \mathrm{day}^{-1}$ was proved to be sufficient. Fourteen days after the beginning of the treatment, however, a dosage increase up to $4 \mathrm{mg} \mathrm{day}^{-1}$ has become necessary for the other seven patients due to poor beneficial effects and an NRS score $\geqslant 3$. During the treatment period, all the patients reported great beneficial effects, based on their own subjective parameters, and NRS scores equal to 1 (Figure 5).

One month after the therapy interruption, subjective beneficial effects were still reported by all patients, although the associated scores ranged between 1 and 2 . Three months after the therapy interruption, four patients reported a score equal to 3 , whereas all the other patients reported a score greater than 3 . None of the patients have shown side effects of the given medicine (Figure 6).

\section{Discussion}

Although the record of cases is limited, it seems evident that the bornaprine treatment, at 2 or $4 \mathrm{mg} \mathrm{day}^{-1}$ dosage, has been effective and safe to control hyperhidrosis in the acute phase of spinal cord-injured patients. The treatment period in the acute phase and the long term, however, are still open questions to be addressed. It would be desirable to confirm these data with trials and a larger number of cases.

\section{References}

1 Andersen LS, Biering-Sorensen F, Muller PG, Jensen IL, Aggerbeck B. The prevalence of hyperhidrosis in patients with spinal cord injuries and an evaluation of the effect of dextropropoxyfene hydrochloride in therapy. Paraplegia 1992; 30: 184-191.

2 Shessel FS, Carrion HM, Politano VA. Phenoxybenzamine and sweating in the spinal cord injury patient. J Urol 1978; 120: 60-61.

3 Tashjian EA, Richter KJ. The value of propoxyfene hydrochloride for treatment of hyperhidrosis in the spinal cord injured patient: an anecdotal experience and case report. Paraplegia 1985; 23: 349-353.

4 Canaday BR, Stanford RH. Propantheline bromide in the management of hyperhidrosis associated with spinal cord injury. Ann Pharmacother 1995; 29: 489-492.

5 Castells Rodellas A, Moragon Gordon M, Ramirez Bosca A. Efecto de la Bornaprina en las hiperhidrosis localizadas. Med Cutan Ibero Lat Am 1987; 15: 303-305. 